\title{
Review of: "Public health vulnerability due to the exposure of dissolved metal(oid)s in tap water from a mega city (Dhaka, Bangladesh)"
}

Jordan Brizi Neris

Potential competing interests: The author(s) declared that no potential competing interests exist.

Major comment:

This study investigated the public health vulnerability associated with dissolved metal(oid)s in 34 household tap-water samples collected in Dhaka city, whose studies on the health risk and tap-water quality are still scarce. The article presents a relevant theme, good writing, detailed and complete methodology, accurate and complete figures and tables, and a good discussion of the results. Nevertheless, a few suggestions and questions about the manuscript are presented below.

1 - It would be very interesting to provide a table (in the supplementary material) with the coordinates of the sampling points.

2 - Did the authors carry out the validation of the analytical method used to quantify the chemical species? Even if the procedure is the same as in other articles, readers of this manuscript must have all the necessary data for validation and interpretation of the presented results. The author must describe briefly the type of validation performed (recovery from standard samples, addition of the chemical species to the matrix, among others) in the methodology section and provide the validation results in the supplementary material.

3 - Across all the manuscript (including Tables and Figures), the unit of measurement ( $\mu \mathrm{g} / \mathrm{L}$ ) of the potentially toxic elements' concentrations must be changed to $\mu \mathrm{g} \cdot \mathrm{L}-\mathrm{l}$ or $\mu \mathrm{g} \mathrm{L}-\mathrm{I}$.

4 - It would be very interesting to describe (at the end of the file) the references cited in the supplementary material. In this way, it is not necessary to go to the principal manuscript file to get the reference description.

5 - In Figures 2 and 4, does the whisker of the mean values indicate the confidence interval? If yes, why show only the positive error?

6 - The comparison analysis of heavy metal concentrations with the national and international 
recommended guideline values performed in section 3.2 is repetitive. I suggest integrating this part with the same type of analysis performed in the first paragraphs of section 3.1.

7 - In line 389, I suggest replacing "heavy metals" with "potentially toxic elements (PTE)" or "chemical species" or others more current and appropriate terms and standardizing this throughout the manuscript. Also, that same line appears to have a typo (thee).

8 - The authors used the RfD of the chemical species $\mathrm{Cr}(\mathrm{VI})$ to perform the human health risk assessment. During the quantification of $\mathrm{Cr}$ concentrations, did the authors perform chemical speciation analysis? If not, why did the authors use the RfD of $\mathrm{Cr}(\mathrm{VI})$ instead of $\mathrm{Cr}(\mathrm{III})$, which has much lower toxicity ( $\mathrm{RfD}=1500$ $\mu \mathrm{g} \cdot \mathrm{kg}-1 \cdot \mathrm{d}-1$, source: EPA-IRIS system)? $\mathrm{Cr}(\mathrm{III})$ is usually the most abundant ion since it forms the most stable compounds and occurs in nature. Therefore, without a confirming speciation analysis, there is a high probability that a majority of the total chromium quantified in this study is $\mathrm{Cr}(\mathrm{III})$, even with the presence of $\mathrm{Cr}(\mathrm{VI})$ contamination sources in the study area. 